\title{
HABRONEMOSE NASAL EM UMA ÉGUA
}

FREITAS, Fernanda Coutinho de ${ }^{1}$ MORAES, Angélica Trazzi Bento de ${ }^{2}$ VALENTE, Paula Pimentel ${ }^{3}$

AGOSTINHO, Juliana Maria Avanci ${ }^{4}$ MAGALHÃES, Geórgia Mode ${ }^{3}$

RESUMO: A Habronemose é uma doença parasitária, e sua patogenia não está totalmente clara. Supõe-se que as larvas mortas ou que estão morrendo, desencadeiem uma reação de hipersensibilidade. Pode-se realizar tanto o tratamento clínico, como a exérese cirúrgica do granuloma caso seja necessária. O presente caso ilustra uma enfermidade das vias aéreas inferiores secundária a uma afecção parasitária atípica obstruindo o tecido nasal e adjacências. A terapia clínica instituída através da aplicação de neguvon intranasal, mostrou-se eficiente, não ocorrendo recidivas.

Palavas-chave: Habronema. Atípica. Equino.

\section{NASAL HABRONEMIASIS IN A MARE}

SUMMARY: The habronemiasis is a parasitic disease and its pathogenesis is not entirely clear. It is assumed that maggots dead or dying, eliciting a hypersensitivity reaction. You can perform both clinical treatment such as surgical excision of the granuloma if needed. This case illustrates a lower airway disease secondary to an atypical parasitic disease by blocking the nasal tissue and surroundings. Therapy clinic established by the application of intranasal neguvon, was efficient, with no recidivism.

Keywords: Habronemosis. Atypical. Equine.

\section{INTRODUÇÃO}

A Ordem Spiruroidea inclui nematóides do gênero Habronema, apresentando duas espécies de importância veterinária, a Habronema muscae e a Habronema microstoma (THOMASSIAN, 2005; LEITÃO, 1983). Trata-se de uma afecção parasitária ocasionada por estes nematóides (ANDRADE, 2002), sendo estas relatadas na forma conjuntival, cutânea e gástrica (SOULSBY, 1965; TREES et al., 1984; MOHAMED et al., 1990; GASTHUY et al., 2004).

O Habronema sp geralmente parasita a porção glandular do estômago de equídeos, muares e asininos ( BERTONE, 2000; FORTES, 2004; URQUHARD et al., 1990 ),

\footnotetext{
${ }^{1}$ Médica Veterinária do Hospital Veterinário da Fafram

${ }^{2}$ Prof. ${ }^{\text {a }}$ Dr ${ }^{\mathrm{a}}$. do Curso de Medicina Veterinária da Fafram

${ }^{3}$ Prof. a Msc do Curso de Medicina Veterinária da Fafram

${ }^{4}$ Aprimoranda do Hospital Veterinário da Fafram
} 
principalmente na região do margo plicatus, medindo cerca de 1 a 2,5 $\mathrm{cm}$ de comprimento (THOMASSIAN, 1997; LEITÃO, 1983); seus ovos são compridos e finos, contendo as larvas (SOULSBY, 1965; BOWMAN; LINN, 1999). A Mosca doméstica e a Stomoxys calcitrans são considerados hospedeiros intermediários deste parasita (FORTES, 1997; BLAGBURN, 1991).

Durante seu ciclo evolutivo, as fêmeas do Habronema sp. fazem ovipostura de ovos embrionados, podendo ocorrer a eliminação destes nas fezes, como também no intestino, através da eclosão das larvas. No ambiente, as larvas L1 são ingeridas por larvas do hospedeiro intermediário ocorrendo o desenvolvimento de ambas concomitantemente. $\mathrm{O}$ hospedeiro intermediário adulto com a larva infectante L3, pode depositar esta em feridas cutâneas do eqüino ou este pode ingerir L3 causando, respectivamente, Habronemose cutânea e Habronemose gástrica (BERTONE, 2000; FORTES, 2004). Quando depositadas sobre uma ferida cutânea ou ao redor dos olhos, as larvas invadem os tecidos, no entanto, não completam seu desenvolvimento (URQUHARD et al., 1990).

O Habronema vive livremente no estômago, em uma camada de muco aderida na mucosa, podendo ou não invadir as glândulas gástricas (FORTES, et al., 1997; BLAGBURN et al., 1991; AIELLO et al., 2001). As manifestações clínicas são raras, podendo ocasionar uma irritação da mucosa gástrica, que irá desencadear uma hipersecreção de muco viscoso e espesso, devido à hiperplasia das células produtoras de muco. Por consequiência ocorre um quadro de gastrite catarral crônica (THOMASSIAN, 2005; BLAGBURN et al., 1991; ROSE et al., 1995). A presença desse parasita no estômago pode agir como fator predisponente ao aparecimento de úlceras gástricas (SILVA et al., 2002). A infecção gástrica é de difícil diagnóstico já que os ovos e as larvas não são detectados facilmente nas fezes através das técnicas de flutuação (FORTES, 1997; URQUHARD et al., 1990; BLAGBURN et al., 1991; ROSE et al., 1995). O Xenodiagnóstico (FORTES, 1997; THOMASSIAN, 2005) e a detecção de ovos e parasitas por lavagem gástrica são possíveis, porém apresentam pouca praticidade e eficiência. Leitão (1983); Aiello et al. (2001); Belli et al. (2005) relataram a gastroscopia como sendo uma técnica fácil e eficiente na detecção da Habronemose gástrica.

De acordo com os relatos encontrados na literatura, o tratamento com a ivermectina na dose $0,2 \mathrm{mg} / \mathrm{kg}$, mostra-se bastante eficaz, sendo descrito por Pérez, et al. (2002) e também a eficácia da administração oral de Doramectina na dose $0,2 \mathrm{mg} / \mathrm{kg}$.

A principal importância clínica do Habronemose reside no fato de suas larvas invadirem os olhos, causando conjuntivite e feridas na pele, induzindo a um quadro de Habronemose cutânea ( THOMASSIAN, 2005; LEITÃO, 1983; ROSE et al, 1995 ). Trata-se 
de uma dermatite granulomatosa, ulcerativa, com múltiplos focos de necrose coagulativa, sendo esta conhecida como "ferida de verão" ou "esponja" ( PAIVA, 1988 ). Estas lesões ocorrem com maior frequiência em locais que apresentam maior ocorrência traumática e onde o animal não consegue remover as moscas como, por exemplo, o rosto, região medial dos olhos, linha média do abdômen, em torno do pênis e prepúcio, além das patas, anca e pescoço. Devido ao intenso prurido, ocorre o autotraumatismo. Forma-se, então, um granuloma castanho avermelhado não cicatrizante, podendo tornar-se, mais tarde, uma lesão fibrosa e inativa (HAMMOND et at., 1986). O granuloma evolui rapidamente, atingindo até grandes diâmetros. Possui o centro côncavo e tecido de granulação irregular (THOMASIAN, 2005).

A patogenia da enfermidade não está totalmente clara, supõe-se que as larvas mortas ou que estão morrendo, desencadeiem uma reação de hipersensibilidade (REED; BAYLY, 2000).

A Habronemose cutânea pode ser diagnosticada pelo encontro e identificação de larvas em raspado de pele ou biópsia da lesão (SNOW et al., 1979; FORTES, 2004). As características histológicas das lesões consistem em dermatite eosinofílica e necrose coagulativa (TRAVERSA et al., 2007; PUSTERLA et al., 2003), apresentando também eosinofilia (VILLA, 1952). A PCR tem sido relatada como um meio diagnóstico da Habronemose gástrica (GIANGASPERO et al., 2005; TRAVERSA et al., 2004) e cutânea (TRAVERSA et al., 2007). Trata-se de um método com sensibilidade apurada e de alta especificidade, permitindo a delineação dos parasitas, independentemente de suas fases do ciclo de vida (TRAVERSA et al., 2004). A aplicação desta técnica melhora o prognóstico de tal infecção parasitária, devido à sua precocidade. Um diagnóstico precoce evita o aparecimento de cicatrizes desfigurantes, o que, quando presentes na glande ou jarretes, podem limitar o garanhão em acasalar com as éguas (TRAVERSA et al., 2009).

O tratamento para a Habronemose cutânea inclui a administração de corticosteróides e combinações tópicas de antiinflamatórios, larvicida e antibióticos (GASTHUYS et al., 2004; PUSTERLA et al., 2003; REBHUN et al., 1981). Em casos de Habronemose conjuntival, o uso de pomada oftálmica é necessária e eficaz (YARMUT et al., 2008). O tratamento cirúrgico deve ser instituído em casos de lesões que não cicatrizam ou em casos de nódulos calcificados que causem transtornos estéticos. Além disso, criocirurgia e radioterapia podem ser usadas (SMITH, 1994). Garcia et al., (2008) relataram o uso de autohemoterapia ozonizada como tratamento eficaz na Habronemose cutânea.

O objetivo do presente estudo foi descrever um caso de broncopneumonia associada à Habronemose atípica, localizada na cavidade nasal, avaliando as medidas terapêuticas instituídas em uma égua sem raça definida. 


\section{RELATO DE CASO}

Foi atendida uma égua sem raça definida, com sete anos de idade, apresentando histórico de corrimento nasal sero-sanguinolento, tosse e queda da performace há cinco meses. $\mathrm{O}$ animal foi tratado na propriedade, há três meses, com penicilina benzatina (44.000 UI, 1 vez ao dia, 5 dias) e Gentamicina (6,6 mg/kg, 1 vez ao dia, 3 dias), apresentando uma melhora clínica com a terapia instituída. Após dois meses do término da terapia clínica, os sinais clínicos apresentados anteriormente foram agravando e o animal foi encaminhado ao Hospital Veterinário. Os exames físicos e diagnósticos como, rinoscopia, histopatológico e hemograma foram realizados. O exame físico evidenciou uma severa intolerância ao exercício, dispnéia, mucosa gengival cianótica, aumento dos linfonodos mandibulares, secreção nasal bilateral mucopurulenta e sero-sanguinolenta, tosse, ruído inspiratório, presença de um granuloma na narina direita (Figura 1), ruído laringotraqueal, presença de estertor creptante bilateral com presença de sibilos na margem dorsal do lobo cranial do pulmão direito e na margem ventral do lobo cranial do pulmão esquerdo. A rinoscopia realizada nas vias aéreas superiores, na região de meatos e cornetos nasais, evidenciou a presença de uma massa granulomatosa (Figura 1) bloqueando totalmente a passagem aérea do lado direito, e a presença de um pequeno granuloma localizado no ducto naso-lacrimal esquerdo. Após a excisão cirúrgica de um pequeno fragmento, o resultado do exame histopatológico relatou abundante quantidade de eosinófilos, com áreas de necrose sugerindo o diagnóstico de Habronemose.

Após a realização do exame físico e exames complementares, suspeitou-se de broncopneumonia secundária a uma habronemose nasal, optando-se pelo tratamento conservador. Foi administrado intra-lesional no granuloma presente na narina direita, uma solução contendo $10 \mathrm{~g}$ de Trichlorfon diluído em $10 \mathrm{ml}$ de água destilada e $5 \mathrm{ml}$ de DMSO, a cada 8 dias (Figura 2), totalizando 4 aplicações (Figura 2). As crostas formadas sobre o granuloma eram removidas e a ferida higienizada diariamente com uma solução de furanil. A seguir, era instilado um aerosol sobre as lesões, contendo $500 \mathrm{mg}$ de sulfato de neomicina e 1 g de hidrocortizona, duas vezes ao dia, até a regressão total do granuloma (Figura 3). Instituiu-se como terapia sistêmica ainti-inflamatória o acetato de isoflupredona, na dose de $0,05 \mathrm{mg} / \mathrm{kg}$, a cada 3 dias, totalizando 4 aplicações. Já na narina esquerda, a terapia tópica não foi necessária, ocorrendo a regressão do pequeno granuloma apenas com a terapia sistêmica. Como terapia broncodilatadora utilizou-se o cloridrato de clembuterol $(0,8 \mu \mathrm{g} / \mathrm{kg}, 2$ vezes ao dia, 20 dias); como terapia expectorante e pneumoprotetora o fluimucil $10 \%(1,5 \mathrm{mg} / \mathrm{kg}, 2$ vezes, 7 dias) e a terapia antimicrobiana com sulfa + trimetropim (10mg/kg, 1 vez, 5 dias). 
Foi administrado ivermectina $1 \%$ pasta, intercalada com moxidectim $(200 \mu \mathrm{g} / \mathrm{Kg}$, a cada 10 dias, totalizando 4 administrações), como terapia anti-helmíntica.

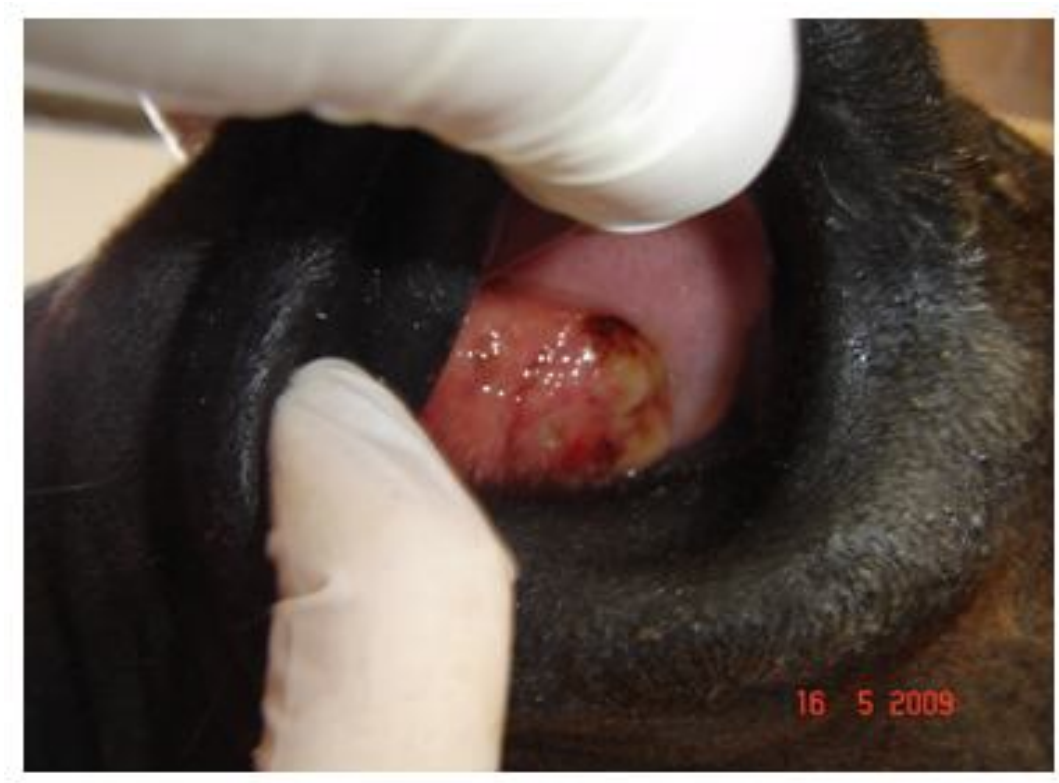

Figura 1- Aspecto inicial do granuloma presente na narina direita.

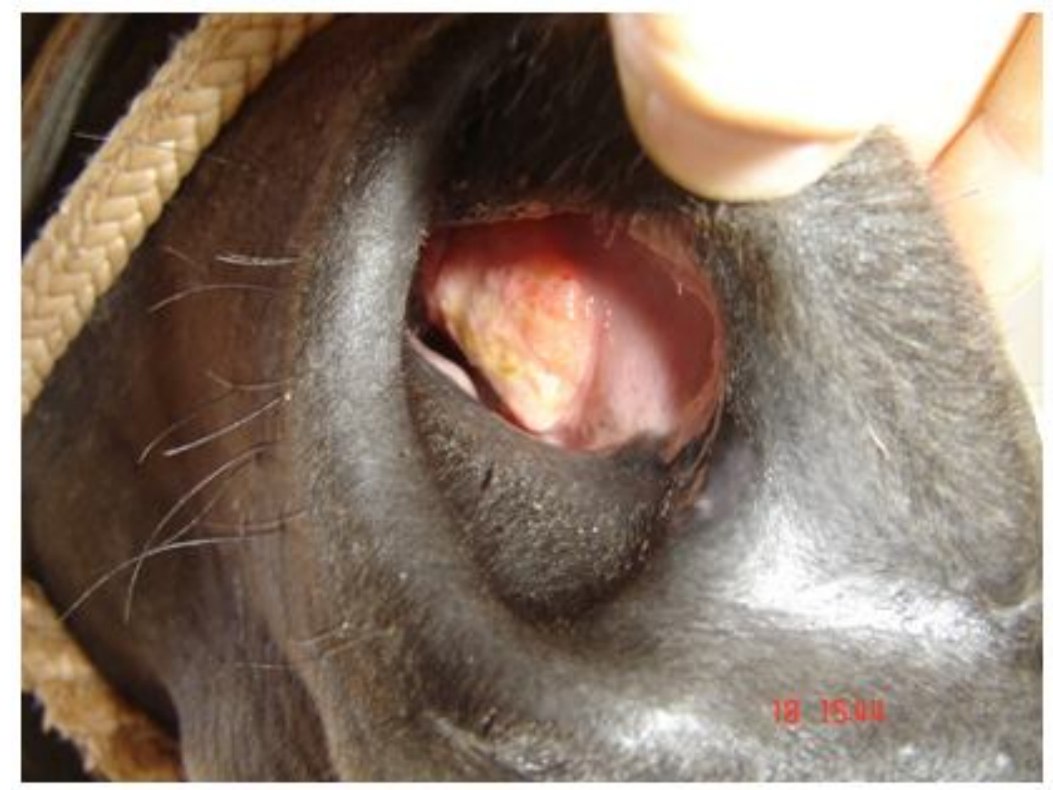

Figura 2- Regressão do granuloma após a primeira administração intra-lesional da solução terapêutica. 


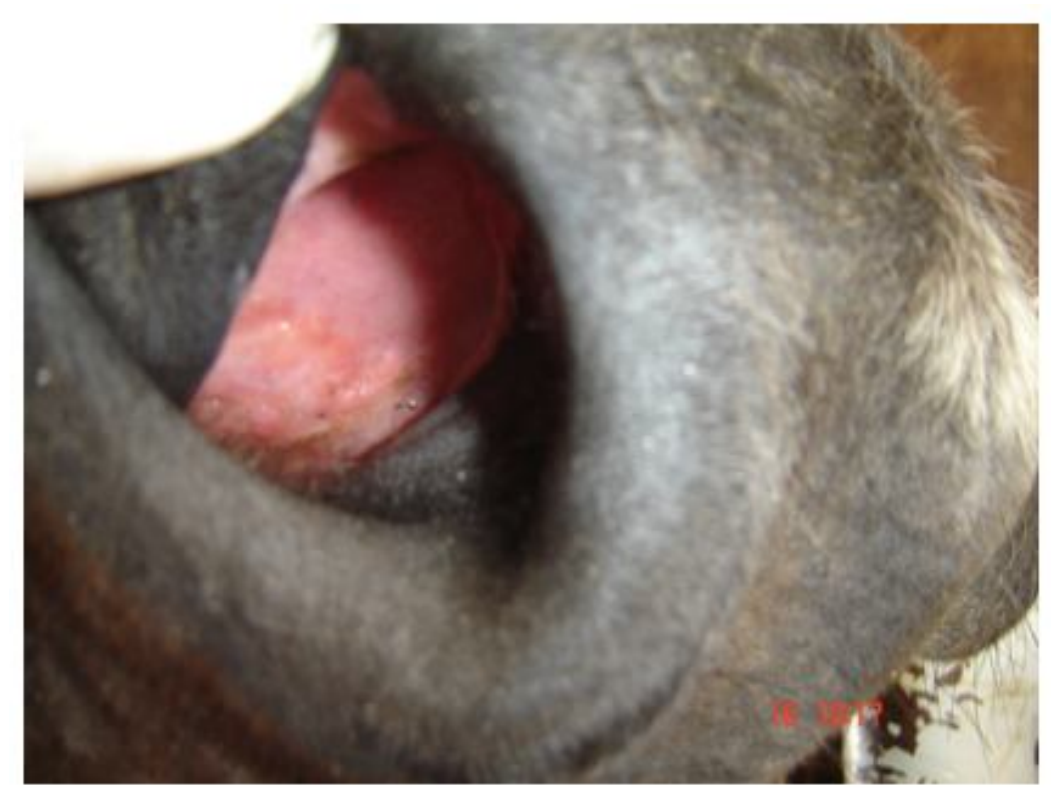

Figura 3- Imagem da regressão total do granuloma após o término do tratamento.

\section{RESULTADOS E DISCUSSÃO}

Embora o agente Habronema sp não tenha sido visualizado no exame histopatológico, o infiltrado eosinofílico foi compatível com o quadro de Habronemose, uma vez que, em colorações especiais como PAS e Grocott, os resultados foram negativos para lesões fúngicas, com finalidade de diagnóstico diferencial.

A administração intra-lesional da solução terapêutica, promoveu uma cauterização local e consecutivamente, uma regressão dos granulomas em um curto período de tempo (Figura 3), no entanto, há indicação sistêmica do Triclorfon diluído em solução salina por via intravenosa, apresentando também resultado satisfatório (SMITH, 1994).

Optou-se pela combinação de dois compostos antiparasitários, ivermectina e moxidectin, em virtude de uma possível resistência parasitária; já que o moxidectin possui efeito moderado contra as larvas encistadas de terceiro e quarto estágio e efeito nulo sobre as larvas em hipobiose (MARTIN, 1997). A ivermectina possui um período de ação de até 3 semanas, eliminando as larvas que entram na pele, mas provavelmente possui uma pequena atuação na regressão do tamanho da lesão original (HERD; DONHAM, 1981).

A terapia sistêmica indicada é a base de glicocorticóides, sendo a prednisolona a mais utilizada, por possuir uma ação mais rápida quando comparado a dexametasona. No entanto, optou-se pela utilização do acetato de isoflupredona por se tratar de um glicocorticóide eficaz e de baixo custo, quando comparado aos demais produtos comercialmente disponíveis; cuja aplicação tem por objetivo reduzir a resposta inflamatória gerada pelo animal em relação ao parasito (SCOTT; MILLER, 2003). 
Os sinais clínicos compatíveis com uma pneumonia cessaram após 15 dias de tratamento e, o granuloma reduziu totalmente com 40 dias de tratamento.

A enfermidade usualmente começa na primavera com o aumento de populações da mosca (DONHAM, 1981), sendo assim, o controle dos parasitas fundamental, pois refletem em melhores condições sanitárias. Preventivamente, as instalações onde os animais vivem, devem ser limpas, eliminando assim os focos de proliferação de moscas (THOMASIAN, 2005).

Alguns métodos de controle do vetor, a moscas adulta devem ser empregados, como a telagem das portas das baias; o pincelamento utilizando produtos tóxicos formulados à base de organofosforados e piretróides, tem se mostrado eficiente por um período de vários dias em animais e estábulos; a utilização de equipamento elétrico contendo lâmpadas com luz azul, como atrativo e a descarga elétrica como agente letal, tem atendido às necessidades de eliminação do adulto.

Como também, o controle das formas jovens, através do manejo adequado dos resíduos dos equinos em confinamento; a utilização de larvicidas na ração; deposição correta do resíduo sólido "lixo" através do aterro controlado ou sanitário (RIO GRANDE DO SUL, 1996; AURVALE; GUAZZZELE, 1986).

Nos criatórios utiliza-se exclusivamente os compostos antiparasitários do grupo das lactonas macrocíclicas, como a ivermectina e moxidectin para controle, em virtude da sua praticidade e eficiência (MONAHAN et al., 1996).

\section{CONCLUSÃO}

Neste relado, a afecção das vias aéreas inferiores foi secundária ao processo obstrutivo das vias aéreas superiores, constituído de granulomas, que foram induzido por uma enfermidade parasitária e se instalaram em uma região atípica. Portanto, esta deve estar inclusa no diagnóstico diferencial das afecções das vias aéreas superiores.

A terapia clínica instituída foi satisfatória, não ocorrendo recidivas após 18 meses do término do tratamento.

\section{REFERÊNCIAS}

AI ELLO, S. E.; MAYS, A. Parasitas gastrointestinais dos eqüinos. In: AIELLO, S. E.; MAYS, A. (Ed.). Manual Merck de veterinária. 8. ed. São Paulo: Roca, 2001. p. 165-170. ANDRADE, S.F. Manual de terapêutica veterinária. 2 ed. São Paulo: Roca, 2002. p.99 112. 
AURVALE, A. E.; GUAZZZELE, M. J. Controle natural de pragas domésticas. 3. ed.Porto Alegre: ADFG - Amigos da Terra. 1986.

BELLI. C. B. et al. Endoscopia na Habronemose gástrica eqüina. Endoscopy in equine gastric habronemosis. Endoscopia en la habronemosis gástrica equiina. Rev. Educ. Contin. CRMVSP/ Contin. Educ. J. São Paulo, CRMV-SP v. 8, n.1, p. 13-18, 2005.

BERTONE, J. J. Prevalence of gastric ulcers in elite, heavy use western performance horses. Proceedings of the 46th Annual AAEP Convention, v.46, 2000.

BITTENCOURT, A. J., BORJA, G. E. M. Stomoxys calcitrans (L.): Preferência por Regiões do Corpo de Eqüinos para Alimentação. Parasitologia al Dia, Santiago de Chile, 2000. p. $119-122$.

BLAGBURN, B. L. et al. Pathogenesis, treatment and control of gastric parasites in horses. Compendium on Continuing Education for the Practicing Veterinarian, v. 13, p. 850857, 1991.

BOWMAN, D.D., LYNN, R.C. Georgis' parasitology for veterinarians. 7 ed. Sauders, Philadelphia, 1999.

FORTES, E. Subfamília Habronematinae. In: FORTES, E. (Ed). Parasitologia veterinária. São Paulo: Ícone, 1997. p.384-391.

FORTES, E. Parasitologia veterinária - 4ed. - São Paulo: Ícone, 2004. P. 342- 348.

GARCIA, C.A.et al. Autohemoterapia maior ozonizada no tratamento de habronemose em eqüino: relato de caso. Disponível em:

<http://www.sovergs.com.br/conbravet2008/anais/cd/resumos/R0608-1.pdf> Acesso em: 10 nov. 2009.

GASTHUY, F.M., VAN HEERDEN, M., VERCRUYSSE, J. Conjunctival habronemiosis in a horse in Belgium. Vet. Rec., v.154, n.24, p.757-758, 2004.

GIANGASPERO, A., TRAVERSA, D.; OTRSNYO, D. A new tool for the diagnosis in vivo of habronemosis in horses. Equine. Vet. J. v.37, n. 263-264, 2005.

HAMMOND, C.J.; MASON, D.K.; WATKINS, K.L. Gastric ulceration in mature Thoroughbred horses. Equine Veterinary Journal, v.18, p.284-287,1986.

HERD, R.P.; DONHAM, J.C. Efficacy of ivermectin against cutaneous Draschia and Habronema infection (Summer sores) in horses. Am J Vet Res. n.42, p. 1953, 1981.

LEITÃO, J. S. Espirurídeos. In: Parasitologia veterinária. 3. ed. Lisboa: Fundação Calaouste Gulbenkian, 1983. p. 156-158.

MAURO, L.F.F.et al. Habronemose Cutânea. Revista Científica de Medicina Veterinária, v.2, n 11, p. , 2008.

MARTIN, R.J. Modes of action of anthelmintic drugs. Veterinary Journal, v.154, p.11-34, 1997. 
MOHAMED, F.H.et al. Cutaneous habronemiasis in horses and domestic donkeys (Equus asinus asinus). Rev. Elev. Med. Vet. Pays. Trop. v.42, n.4, p. 535-540, 1990.

PAIVA, F. Descrição de formas imaturas, uma nova técnica de diagnóstico e a prevalência de habronemíase gástrica no Mato Grosso do Sul. 58f. 1988. Dissertação (Mestrado), Universidade Federal Rural do Rio de Janeiro, Itaguaí.

PEREZ, R. CABEZAS et al. Pharmacokinetics of doramectin and ivermectin after oral administration in horses. The Veterinary Journal 2002, p.163, 161-167.

PUSTERLA, N.et al. Cutaneous and ocular habronemiasis in horses: 63 cases (1988-2002). J. Am. Vet. Med. Assoc. v.222, p. 978-982, 2003.

REBHUN, W. C.et al. Habronemic Blepharoconjunctivitis in horses. J. Am. Vet. Med. Assoc. v.179, p. 469-472, 1981.

REED, S. M.;BAYLY, W. M. Causas parasitárias de nódulos. In: MEDICINA INTERNA EQUINA. Rio de Janeiro: Guanabara Koogan, 2000. p. 461.

RIO GRANDE DO SUL. Secretaria da Saúde e do Meio Ambiente. Departamento de Ações de Saúde. Seção de Zoonoses e Vetores. Programa de Controle de Moscas Sinantrópicas Normas Técnicas Operacionais. 1996.

ROSE, R. J.; HODGSO ,D. R. Sistema alimentario. In: O MANUAL clínico de equinos. México: Interamericana-McGraw-Hill, 1995. p. 232-30 I.

SILVA, L. C. L. C.; ALVARE GA, J.; KLEMM, M. Efeitos de drogas anti inflamatórias não esteroidais e estresse sobre a mucosa gástrica de eqüinos. Brazilian Journal of Veterinary Science, v. 9, p. 92-94, 2002.

SCOTT, D.W.; MILLER, W.H. Jr. Equine dermatology. St Louis: Saunders, 2003. 823p.

SMITH, B.P. Tratado de medicina interna de grandes animais. São Paulo: Manole, v.2, 1994.

SNOW, D.H. et al. Phenylbutazone toxicity in ponies. Vet. Rec. v.105, p.26-30, 1979.

SOULSBY, E.J.L. Text book of veterinary clinical parasitology. Helminths, Blackwell, Oxford, 1965.

TRAVERSA, D.et al. Molecular diagnosis of equid summer sores. Vet. Parasitol. v.150, p. 116-121, 2007.

TREES A.J.; MAY, S.A.; BAKER, J.B. Apparent case of equine cutaneous habronemiasis. Vet. Rec. v.115, p.1, p.14-15, 1984.

THOMASSIAN, A. Parasitos do estômago e intestinos. In: ENFERMIDADES dos cavalos. 2. ed. São Paulo: Varela, 2005. p.176.

URQUHARD, G. M. et aI. Helmintologia veterinária. Parasitologia veterinária. Rio de Janeiro: Guanabara Koogan, 1990. p.3-145. 
VILLA, E. Contribución al Estúdio de la Habronemosis cutânea (Valor del Hemograma em el Diagnóstico). Tesis Bach. Med. Vetr. UNMSM. 137p., 1952.

YARMUT, Y. et al. Ophthalmic and cutaneous habronemiasis in a horse: case report and review of the literature. Israel Journal of Veterinary Medicine, v. 63, 2008. 